\title{
Actitud de los pediatras sobre el uso de andadores para los bebés
}

\author{
Pediatricians' attitude about the use of infant walkers
}

\author{
Dra. Semra Kara ${ }^{a}$, Dra. Ayşe Esra Yılmaz ${ }^{a}$ Dra. Derya Gümüşs Dogan ${ }^{a}$, Dr. Meki Bilici ${ }^{a}$, Dr. Sadi Turkay ${ }^{a}$, \\ Dra. Halise Akca y Dr. Ferhat Catal
}

\begin{abstract}
RESUMEN
Los andadores se utilizan frecuentemente a pesar de que su uso puede ocasionar lesiones. Se realizó una encuesta para obtener información sobre las actitudes y el abordaje de los pediatras respecto del uso de andadores. Se invitó a 247 pediatras que asistieron al $44^{\circ}$ Congreso Turco de Pediatría y Europediatría (2008) a responder un cuestionario preparado por los autores. Se incluyeron en el análisis 226 cuestionarios respondidos en forma completa.

La mediana de edad de los participantes (119 mujeres) fue de 39 años (29 a 58). De ellos, $4 \%$ recomendaban el uso de andadores, 32,3\% dejaban la decisión a criterio de los padres y $63,7 \%$ no lo recomendaban. Ciento cinco habían tratado con anterioridad a un bebé que sufrió una lesión relacionada con el uso del andador; de ellos, 73,3\% no recomendaron su uso y $57,1 \%$ opinaron que la producción y el uso de andadores deberían prohibirse.

Conclusiones. El $4 \%$ de los pediatras encuestados recomiendan el uso de andadores para bebés y más del 30\% dejan la decisión a los padres. Los pediatras que atendieron a un bebé que sufrió una lesión por el uso del andador fueron menos proclives a recomendarlo.

Palabras clave: andador, pediatra, prevención de las lesiones infantiles.
\end{abstract}

http:/ /dx.doi.org/10.5546/aap.2013.528

\section{INTRODUCCIÓN}

Los andadores son todavía de uso común en todo el mundo, aunque pueden ocasionar accidentes domésticos, quemaduras y envenenamientos. ${ }^{1,2}$ Los padres pueden considerarlos seguros porque mantienen al bebé tranquilo y satisfecho, le permiten ejercitarse y caminar antes, favorecen su movilidad y le proporcionan un lugar de alimentación, al tiempo

a. Departamento de Pediatría, Fatih University, Faculty of Medicine, Ankara, Turquía.

\section{Correspondencia: \\ semrakara@hotmail.com}

Conflicto de intereses: Ninguno que declarar.

Recibido:24-12-2012

Aceptado: 29-7-2013 que le brindan a la madre la oportunidad de hacer las tareas domésticas. ${ }^{2-5}$ Aunque la tasa de uso de andadores para bebés varía en todo el mundo, las informadas son muy altas: $50 \%$ en el Reino Unido, ${ }^{3} 70 \%$ a $90 \%$ en los Estados Unidos, ${ }^{6,7}$ $55 \%$ en Dublín, Irlanda, ${ }^{8} 54,5 \%$ en Irán, ${ }^{9} 90 \%$ en Singapur ${ }^{4}$ y $75,4 \%$ en Turquía. ${ }^{1}$ Los andadores están asociados con lesión en el $12 \%$ a $40 \%$ de los niños que los utilizan. ${ }^{3,4,10,11}$ Más aún, se informó que su uso causó la muerte de 34 bebés en los Estados Unidos entre 1973 y $1998 .{ }^{11}$ Esas muertes suelen relacionarse con un traumatismo craneal sufrido al caerse por una escalera. ${ }^{11} \mathrm{El}$ uso de andadores aumenta el riesgo de lesión por caída de escalera cuatro veces y el riesgo de fractura por esta caída, dos veces. ${ }^{12}$ Debido a estos riesgos, la Academia Estadounidense de Pediatría propone prohibir la producción y venta de andadores para bebés, ${ }^{13}$ como sucede en Canadá desde $2004 .{ }^{14}$

El $25 \%$ a $50 \%$ de los padres cuyos hijos sufrieron una lesión relacionada con el andador lo han seguido utilizando; es necesario que los pediatras orienten a los padres de un modo más firme y contundente. ${ }^{15-17}$ En nuestro conocimiento, hay pocos estudios sobre la actitud de los pediatras al respecto. El objetivo del presente estudio fue obtener información acerca de las actitudes de los pediatras sobre el uso de andadores y el abordaje de este tema con los padres.

\section{MATERIAL Y MÉTODOS}

El estudio incluyó a pediatras que asistieron al $44^{\circ}$ Congreso Turco de Pediatría celebrado en Estambul en junio de 2008. Después de informar a todos los asistentes sobre el estudio, se les pidió que respondieran a un cuestionario de 23 preguntas preparado por los autores. Se registró la edad, el sexo, el grado, la afiliación, las actitudes respecto del uso del andador, la lectura de artículos científicos sobre el tema, la experiencia en el tratamiento de pacientes con lesiones asociadas a ese uso, y las actitudes hacia la producción y venta de andadores para bebés. El estudio fue aprobado por el Comité de Ética de 
la Facultad de Medicina de la Universidad Fatih.

Los datos fueron analizados mediante el paquete estadístico para las Ciencias Sociales (SPSS Inc., Chicago, IL, EE.UU.), versión 13,0. Para la comparación de variables categóricas pareadas o múltiples se utilizó la prueba de la $\chi^{2}$. Se consideró significativo un valor de $p<0,05$.

\section{RESULTADOS}

Se invitó a participar a 247 pediatras. Los formularios con respuestas incompletas se excluyeron $(n=21)$. Se analizaron los formularios correspondientes a 226 pediatras. La mediana de edad de los participantes (107 hombres y 119 mujeres) fue de 39 años (29 a 58). Las características demográficas de los participantes se presentan en la Tabla 1.

Las respuestas al cuestionario se detallan en la Tabla 2.

Los pediatras que atendieron a un bebé que sufrió una lesión relacionada con el uso del
TABLA 1. Características demográficas de los pediatras participantes

\begin{tabular}{|c|c|}
\hline Características & \\
\hline Edad, años & 39 \\
\hline \multicolumn{2}{|l|}{ Sexo } \\
\hline Femenino & $119(52,6)$ \\
\hline \multicolumn{2}{|l|}{ Cargo } \\
\hline Residente & $56(24,8)$ \\
\hline Especialista & $138(61,1)$ \\
\hline Profesor asistente & $16(7,1)$ \\
\hline Profesor asociado & $12(5,3)$ \\
\hline Profesor & $4(1,8)$ \\
\hline \multicolumn{2}{|l|}{ Filiación } \\
\hline Sector privado & $76(33,6)$ \\
\hline Ministerio de Salud & $97(42,9)$ \\
\hline Hospital universitario & $53(23,5)$ \\
\hline
\end{tabular}

Datos presentados como mediana \pm desviación estándar o n (\%), cuando corresponde.

TABla 2. Actitudes y abordaje de los pediatras en relación con el uso de andadores

\begin{tabular}{|c|c|}
\hline Preguntas relativas a actitudes y abordaje por parte de los pediatras & n $(\%)$ \\
\hline \multicolumn{2}{|l|}{ ¿Recomienda a los padres el uso de andadores para los niños? } \\
\hline Sí & $9(4)$ \\
\hline Deja la decisión a los padres luego de dar una explicación & $73(32,3)$ \\
\hline No & $144(63,7)$ \\
\hline \multicolumn{2}{|l|}{ Si recomienda el uso de andadores, ¿a partir de qué edad? } \\
\hline 6 meses & $19(23,2)$ \\
\hline 8 meses & $41(50)$ \\
\hline 10 meses & $19(23,2)$ \\
\hline 12 meses & $3(3,6)$ \\
\hline \multicolumn{2}{|c|}{ ¿Deberían los padres ser informados sobre el uso de andadores durante las visitas de control de salud? } \\
\hline Sí & $212(93,8)$ \\
\hline No & $14(6,2)$ \\
\hline \multicolumn{2}{|l|}{ ¿Lee artículos científicos sobre andadores? } \\
\hline Sí & $80(35,4)$ \\
\hline No & $146(64,6)$ \\
\hline \multicolumn{2}{|c|}{ ¿Alguna vez ha tratado a un bebé por una lesión relacionada con el uso del andador? } \\
\hline Sí & $105(46,5)$ \\
\hline No & $121(53,5)$ \\
\hline \multicolumn{2}{|l|}{ Si usted tiene algún hijo, ¿ha usado su bebé un andador? } \\
\hline Sí & $73(47,7)$ \\
\hline No & $80(52,3)$ \\
\hline \multicolumn{2}{|l|}{ ¿Cree que la producción y el uso de andadores deberían prohibirse? } \\
\hline Sí & $91(40,3)$ \\
\hline No & $135(59,7)$ \\
\hline \multicolumn{2}{|c|}{ ¿Piensa que informar a los padres sobre los riesgos del uso del andador disminuirá significativamente su uso? } \\
\hline Sí & $204(90,3)$ \\
\hline No & $22(9,7)$ \\
\hline
\end{tabular}


andador apoyan, en mayor proporción que el resto, la prohibición de la producción y venta de andadores (57,1\% contra $24,8 \%, p<0,001$, Tabla 3$)$.

Con respecto a la pregunta: " ¿Recomienda a los padres utilizar un andador?", cuando se compararon los pediatras que recomiendan el uso frente a los que dejan la decisión a los padres, se encontró una diferencia significativa en haber tratado previamente a un bebé con una lesión causada por el uso de andador $(p=0,016)$. Entre quienes dejan la decisión a los padres, la tasa de los que no habían tratado previamente ningún caso fue del $67,1 \%$.
Entre quienes no recomiendan el uso, la tasa de los pediatras que atendieron a un bebé con esta lesión fue del $53,5 \%$. Por otra parte, no hubo diferencias significativas entre los grupos en cuanto al sexo, la lectura de artículos científicos sobre los andadores y la afiliación (Tabla 4).

\section{DISCUSIÓN}

En el presente estudio, el 63,7\% de los pediatras no recomiendan el uso de andadores y el 32,3\% dejan la decisión a criterio de los padres luego de brindar la explicación pertinente. Aunque solo el $4 \%$ de los pediatras participantes

TABLA 3. Abordaje de los pediatras que trataron previamente a un bebé por una lesión asociada al uso de andador

\begin{tabular}{|c|c|c|c|}
\hline & \multirow{2}{*}{\multicolumn{2}{|c|}{$\begin{array}{l}\text { ¿Alguna vez trató a un bebé por una } \\
\text { lesión asociada al uso de andador? }\end{array}$}} & \multirow{3}{*}{$p$} \\
\hline & & & \\
\hline & Sí n (\%) & No n $(\%)$ & \\
\hline \multicolumn{4}{|c|}{ La producción y el uso de andadores } \\
\hline Deberían prohibirse & $60(57,1)$ & $30(24,8)$ & $<0,001$ \\
\hline No deberían prohibirse & $45(42,9)$ & $91(75,2)$ & \\
\hline Total & $105(100)$ & $121(100)$ & \\
\hline
\end{tabular}

TABla 4. Distribución de respuestas de los pediatras a la pregunta “¿Recomienda el uso de andadores?" de acuerdo con distintos parámetros

\begin{tabular}{|c|c|c|c|c|}
\hline & \multicolumn{3}{|c|}{ ¿Recomienda el uso de andadores? } & \multirow[b]{2}{*}{$p$} \\
\hline & $\begin{array}{l}\text { Recomienda } \\
\qquad \begin{array}{c}(\mathrm{n}=9) \\
\mathrm{n}(\%)\end{array}\end{array}$ & $\begin{array}{c}\text { Deja la decisión a } \\
\text { los padres }(n=73) \\
\text { n }(\%)\end{array}$ & $\begin{array}{l}\text { No lo recomienda } \\
\qquad \begin{array}{c}(\mathrm{n}=144) \\
\mathrm{n}(\%)\end{array}\end{array}$ & \\
\hline \multicolumn{5}{|c|}{ Trató a un bebé con lesión asociada al uso de andador } \\
\hline Sí (n=105) & $4(3,8)$ & $24(22,8)$ & $77(73,3)$ & 0,016 \\
\hline No $(n=121)$ & $5(4,1)$ & $49(40,4)$ & $67(55,3)$ & \\
\hline \multicolumn{5}{|l|}{ Sexo } \\
\hline Masculino $(n=107)$ & $6(5,6)$ & $34(31,7)$ & $67(62,6)$ & NS \\
\hline Femenino $(n=119)$ & $3(2,5)$ & $39(32,7)$ & $77(64,7)$ & \\
\hline \multicolumn{5}{|c|}{ Leyó artículos científicos sobre andadores } \\
\hline Sí $(\mathrm{n}=81)$ & $3(3,7)$ & $21(25,9)$ & $57(70,3)$ & NS \\
\hline No $(n=145)$ & $6(4,1)$ & $52(35,8)$ & $87(60)$ & \\
\hline \multicolumn{5}{|l|}{ Filiación } \\
\hline Sector privado $(n=76)$ & $3(3,9)$ & $26(34,2)$ & $47(61,8)$ & NS \\
\hline Ministerio de Salud $(n=97)$ & $4(4,1)$ & $28(28,8)$ & $65(67)$ & \\
\hline Hospital universitario $(n=53)$ & $2(3,7)$ & $19(35,8)$ & $32(60,3)$ & \\
\hline
\end{tabular}

NS: no significativo. 
de este estudio lo recomiendan, la alta tasa de uso $(75,4 \%)$ en Turquía ${ }^{1}$ sugiere que un número significativo de padres ignoran o hacen caso omiso a los riesgos, o que los pediatras no logran orientarlos de un modo convincente. A pesar de las advertencias sobre los andadores, su uso y las lesiones relacionadas continúan, por lo que las autoridades han propuesto prohibir su producción y venta. Canadá fue el primer país del mundo en hacerlo. ${ }^{14}$

En Francia, Claudet y cols. ${ }^{18}$ evaluaron 178 ingresos hospitalarios que se produjeron durante 2 años debidos a lesiones relacionadas con el uso del andador en un departamento de emergencias pediátricas. El 78\% de estos niños cayeron por la escalera. El autor concluye que el uso del andador debería prohibirse en Francia porque es inseguro y peligroso.

En otro estudio realizado en Grecia de 44/1000 caídas que se produjeron por año, 9 por cada 1000 fueron ocasionadas por el andador y se asociaron a caídas por escaleras y eventualmente a lesiones graves. ${ }^{19}$ Aunque los andadores se utilizan menos por las advertencias y prohibiciones, sus consecuencias continúan existiendo. ${ }^{20-22}$ Desapriya y cols. ${ }^{23}$ refieren que la prohibición de los andadores no es por sí misma suficiente para evitar lesiones; también deberían centrarse los esfuerzos en lograr una concientización de la población. Estos autores sugieren que los padres deben ser informados sobre los andadores, especialmente sobre el hecho de que su uso no es beneficioso para el desarrollo de los lactantes.

Además de los padres, todos aquellos que participan en el cuidado y desarrollo de los niños deberían recibir información al respecto. Distintos estudios han demostrado que su nivel de conocimiento no es suficiente y que se necesitan conocimientos basados en evidencias. ${ }^{24,25}$ Kendrick y cols. ${ }^{25}$ evaluaron las actitudes y conocimientos de los agentes sanitarios sobre el tema e informaron que el $45,8 \%$ de ellos estaban de acuerdo con la prohibición, 13,1\% pensaban que el uso de andadores era beneficioso, $67,2 \%$ opinaban que podría retrasar el comienzo de la marcha y solo $1,7 \%$ creían que los andadores modernos eran seguros para los niños. También concluyeron que la mayoría de los agentes de salud tenían un conocimiento limitado del tema y no discutían con los padres sobre la frecuencia de las lesiones asociadas a su uso. Por lo tanto, sugieren que si el conocimiento de los agentes de salud y parteras se incrementara, la puesta en común de conocimientos con los padres aumentaría. Estos mismos autores informaron resultados posteriores a la formación de agentes de salud y parteras, y concluyeron que las capacitaciones sobre prevención de lesiones se asociaron con un mayor conocimiento, con más actitudes negativas hacia los andadores y con una actitud más positiva hacia la educación de padres y familias respecto de este tema. Las parteras capacitadas eran más propensas a dar consejos antes del parto. ${ }^{26}$

En nuestro conocimiento, solo Rhodes y cols. ${ }^{27}$ evaluaron las actitudes de los pediatras sobre los andadores. Encontraron que un mayor conocimiento se asociaba con actitudes negativas frente a ellos. En el presente estudio, 90,3\% de los pediatras opinaron que informar a los padres reduciría significativamente el riesgo de lesiones asociadas a su uso; sin embargo, el porcentaje de pediatras que leyeron alguno de los artículos científicos sobre los andadores fue de solo $35,4 \%$.

El $47,7 \%$ de los pediatras utilizan un andador para sus hijos. Esta tasa fue de $36 \%$ en el estudio de Rhodes y cols. ${ }^{27}$ Mientras que el 63,7\% de los pediatras no recomiendan el uso de andadores en nuestro estudio, Rhodes y cols. ${ }^{27}$ informaron que el $71 \%$ de los pediatras aconsejaron a los padres no utilizarlos. En ese estudio en particular, 74,1\% de los pediatras también creían que la educación de los padres sobre los riesgos era probablemente más beneficiosa que prohibir su venta.

Por otra parte, "el conocimiento sobre andadores y las lesiones relacionadas" resultó ser un factor que se asoció a quienes siempre dan consejos sobre andadores. En este estudio, los pediatras que atendieron a un bebé por una lesión asociada al andador fueron menos propensos a recomendar su uso. A la luz de los hechos mencionados, consideramos que la reducción del uso de andadores para bebés, que es una importante causa de lesiones infantiles, puede lograrse brindando a los padres conocimiento basado en la evidencia, por parte de los pediatras y los agentes de salud, y también mediante la prohibición de su producción y venta.

Como limitaciones, en nuestro estudio se utilizó un cuestionario autoadministrado que podría dar lugar a un sesgo. Además, los participantes fueron los pediatras que asistieron al congreso celebrado en Estambul. Así, los resultados del presente estudio deben considerarse con cautela y no pueden generalizarse. 


\section{CONCLUSIONES}

En esta muestra, solo el $4 \%$ de los pediatras recomiendan el uso de andadores. Por otro lado, la mitad de los pediatras que tuvieron un hijo lo utilizaron. Los pediatras que atendieron a un bebé con una lesión causada por el uso del andador fueron menos proclives a recomendarlo.

\section{BIBLIOGRAFÍA}

1. Dogan DG, Bilici M, Yilmaz AE, Catal F, Keles N. Baby walkers: a perspective from Turkey. Acta Pædiatr 2009; 98(10):1656-60.

2. Kendrick D, Marsh P. Baby walkers: prevalence of use and relationship with other safety practices. Inj Prev 1998; 4(4):295-8.

3. Thein MM, Lee J, Tay V, Ling SL. Infant walker use, injuries, and motor development. Inj Prev 1997;3(1):63-6.

4. Bar-on ME, Boyle RM, Endriss EK. Parental decisions to use infant walkers. Inj Prev 1998;4(4):299-301.

5. Stoffman JM, Bass MJ, Fox AM. Head injuries related to the use of baby walkers. Can Med Assoc J 1984;131(6):573-5.
6. MarcellaS,McDonaldB.Theinfantwalker:anunappreciated household hazard. Conn Med 1990;54(3):127-9.

7. Use of infant walkers. Board of Trustees, American Medical Association. Am J Dis Child 1991;145(8):933-4.

8. Laffoy M, Fitzpatrick P, Jordan M, Dowdall D. Attitudes to and use of baby walkers in Dublin. Inj Prev 1995;1(2):109-11.

9. Shiva F, Ghotbi F, Yavari SF. The use of baby walkers in Iranian infants. Singapore Med J 2010;51(8):645-9.

10. Kendrick D, Ilingworth R, Woods A, et al. Promoting child safety in primary care: a cluster randomized controlled trial to reduce baby walker use. Br J Gen Pract 2005;55(517):582-8.

11. American Academy of Pediatrics. Committee on Injury and Poison Prevention. Injuries associated with infant walkers. Pediatrics 2001;108 (3):790-2.

12. Khambalia A, Joshi P, Brussoni M, Raina P, etal. Risk factors for unintentional injuries due to falls in children aged 0-6 years: a systematic review. Inj Prev 2006;12(6):378-81.

13. MackKA, GilchristJ, Ballesteros MF. Injuries among infants treated in emergency departments in the United States, 2001-2004. Pediatrics 2008;121(5):930-7.

14. Health Canada. Injury data analysis leads to baby walker ban. [Consulta marzo 2013]. Disponible en: http:/ / www. hc-sc.gc.ca/sr-sr/activ/consprod/baby-bebe-eng.php.

15. Atkinson CC. Another baby walker injury victim. Journal of Emergency Nursing 1997;23(4):302-5.

\title{
Caracterización de mutaciones en el gen GATA-1 en pacientes con Síndrome de Down y diagnóstico de mielopoyesis anormal transitoria o leucemia megacarioblástica aguda Mutation characterization in the GATA-1 gene in patients with Down's Syndrome diagnosed with transient abnormal myelopoiesis or acute megakaryoblastic leukemia
}

\author{
Bioq. Adrián P. Mansini ${ }^{a}$, Bioq. Patricia L. Rubio ${ }^{a}$, Bioq. Jorge G. Rossi ${ }^{b}$, Dra. Marta S. Gallegoc, \\ Téc. Adriana Medina ${ }^{a}$, Med. Pedro A. Zubizarreta ${ }^{a}$, Med. María S. Felice y Bioq. Cristina N. Alonso ${ }^{a}$
}

\section{RESUMEN}

Los pacientes con síndrome de Down tienen un riesgo más elevado de presentar leucemia megacarioblástica aguda (LMCA). Un $10 \%$ de los recién nacidos con ese síndrome

a. Servicio de Hematología y Oncología.

b.Servicio de Inmunología y Reumatología.

c. Servicio de Genética.

Hospital de Pediatría SAMIC "Prof. Dr. Juan P. Garrahan".

Correspondencia: Bioq.Adrián Mansini:adrian_cell@hotmail.com

Este trabajo fue financiado por la Agencia Nacional de Promoción Científica y Tecnológica (FONCyT) y por el Hospital de Pediatría S.A.M.I.C. “Prof. Dr. Juan P. Garrahan”

Conflicto de intereses: Ninguno que declarar.

Recibido: 18-2-2013

Aceptado: 11-6-2013 presentan un cuadro de mielopoyesis anormal transitoria (MAT), indistinguible de la LMCA, que en general remite espontáneamente. En ambos grupos de pacientes se describió una alta incidencia de mutaciones en el gen GATA-1.

Se analizaron 14 muestras de ADN de médula ósea (10 MAT / 4 LMCA) correspondientes a 13 pacientes con Síndrome de Down mediante PCR y secuenciación, para describir la frecuencia y las características de las mutaciones en el gen GATA-1 en la población estudiada y sus consecuencias a nivel proteico. Se detectaron mutaciones en 10 de 10 MAT y en 3 de 4 LMCA, que a nivel proteico originarían un codón de terminación prematuro $(n=5)$, alteraciones en el sitio de corte y empalme (splicing) $(n=6)$ o cambio de secuencia $(n=3)$. Se confirmó la alta frecuencia de mutaciones en el gen GATA-1 en recién nacidos con Síndrome de Down y MAT o LMCA.

Palabras clave: mielopoyesis anormal transitoria, leucemia megacarioblástica, GATA-1, síndrome de Down.

http:/ /dx.doi.org/10.5546/aap.2013.532 


\section{INTRODUCCIÓN}

Los niños con Síndrome de Down tienen una mayor predisposición a padecer leucemia aguda, más frecuentemente leucemia megacarioblástica aguda (LMCA), sobre todo en los dos primeros años de vida. ${ }^{1,2}$ Además, durante el período neonatal, un $10 \%$ de los niños con trisomía 21 presentan un trastorno clonal denominado mielopoyesis anormal transitoria (MAT), indistinguible de la LMCA que, en la mayoría de los casos $(70 \%$ a $80 \%)$, suele resolverse espontáneamente dentro de los 3 meses de evolución. Sin embargo, el $20 \%$ a $30 \%$ restante desarrollan LMCA, en especial durante los 3 primeros años de vida. ${ }^{3}$

El gen GATA binding protein 1 (GATA-1) codifica un factor de activación de la transcripción de $50 \mathrm{kDa}$ que resulta crítico para el normal desarrollo de las ontogenias eritroide y megacariocítica. ${ }^{4,5}$ Recientemente, se describieron mutaciones en el exón 2 de GATA-1 en niños con Síndrome de Down ya MAT o LMCA, que no son detectables cuando la enfermedad se encuentra en remisión. ${ }^{6}$ Se propone que estas mutaciones participan en la evolución de MAT a LMCA en los niños con Síndrome de Down ya que no aparecen en los niños con Síndrome de Down y otros tipos de leucemia aguda ni en los pacientes con LMCA pero sin Síndrome de Down .7,
En el presente estudio, se reportan los resultados del estudio de las mutaciones en el gen GATA-1 en los pacientes con Síndrome de Down y MAT/LMCA ingresados en nuestra institución.

\section{OBSERVACIONES}

Entre 2003-2012 fueron diagnosticados y tratados en nuestro servicio 18 pacientes con diagnóstico de Síndrome de Down y MAT o LMCA. Para el presente estudio se dispuso de 14 muestras de médula ósea (10 MAT/4 LMCA), incluidas dos muestras de un paciente que presentó MAT y luego LMCA.

El exón 2 de GATA-1 y las secuencias intrónicas flanqueantes fueron analizados por PCR y secuenciación. ${ }^{9}$ Los datos clínicos y de laboratorio de los pacientes con Síndrome de Down y MAT o LMCA en el momento del diagnóstico se detallan en la Tabla 1.

Las medianas y rangos de edad de las poblaciones estudiadas fueron 9 ( 1 a 41) días para los pacientes con MAT y 18 (9 a 27) meses para los pacientes con LMCA. Un paciente con MAT desarrolló LMCA 9 meses después de haber alcanzado la remisión completa espontánea de MAT (casos 6 y 12).

Los resultados de los estudios citogenéticos por bandeo-G se muestran en la Tabla 2. En el paciente con MAT que presentó LMCA se

TABla 1. Datos clínicos y de laboratorio de los pacientes con síndrome de Down y mielopoyesis anormal transitoria o leucemia megacarioblástica en el momento del diagnóstico

\begin{tabular}{|c|c|c|c|c|c|c|c|c|}
\hline Caso & Edad* & Diag & Sexo & WBC $\left(\times 10^{9} / \mathrm{L}\right)$ & $\mathrm{Hb}(\mathrm{g} / \mathrm{dL})$ & $\operatorname{Plq}\left(x 10^{9} / \mathrm{L}\right)$ & Hepatomegalia & Esplenomegalia \\
\hline 1 & $4 \mathrm{~d}$ & MAT & M & 45 & 15,1 & 25 & Sí & Sí \\
\hline 2 & $22 \mathrm{~d}$ & MAT & F & 35,1 & 9,8 & 55 & Sí & Sí \\
\hline 3 & $13 \mathrm{~d}$ & MAT & M & 120 & 16,5 & 186 & Sí & Sí \\
\hline 4 & $9 \mathrm{~d}$ & MAT & M & 83,6 & 18,8 & 56 & Sí & Sí \\
\hline 5 & $1 \mathrm{~m}$ & MAT & $\mathrm{F}$ & 8,4 & 9,3 & 23 & Sí & Sí \\
\hline $6^{* *}$ & $6 \mathrm{~d}$ & MAT & M & 47,2 & 16,3 & 195 & Sí & No \\
\hline 7 & $40 \mathrm{~d}$ & MAT & M & 57 & 8,7 & 144 & Sí & Sí \\
\hline 8 & $9 \mathrm{~d}$ & MAT & M & 30 & 12,3 & 102 & Sí & Sí \\
\hline 9 & $1 \mathrm{~d}$ & MAT & $\mathrm{F}$ & 40 & 8,5 & 531 & Sí & Sí \\
\hline 10 & $5 \mathrm{~d}$ & MAT & M & 34,9 & 15,5 & 44 & Sí & Sí \\
\hline 11 & $9 \mathrm{~m}$ & LMCA & F & 6,9 & 10,2 & 84 & Sí & No \\
\hline $12^{* *}$ & $11 \mathrm{~m}$ & LMCA & M & 4,7 & 7,5 & 18 & No & No \\
\hline 13 & $25 \mathrm{~m}$ & LMCA & M & 9,3 & 4,4 & 23 & Sí & Sí \\
\hline 14 & $27 \mathrm{~m}$ & LMCA & F & 25,4 & 3,7 & 10 & Sí & Sí \\
\hline
\end{tabular}

WBC: recuento leucocitario. ${ }^{*}$ Edad: d (días), $\mathrm{m}$ (meses). ${ }^{* *}$ Mismo paciente.

MAT: mielopoyesis anormal transitoria. LMCA: leucemia megacarioblástica aguda. 
534 / Arch Argent Pediatr 2013;111(6):528-536 / Comunicaciones breves

TABla 2. Resultado de los estudios citogenéticos de los 13 pacientes con Síndrome de Down y mielopoyesis anormal transitoria o leucemia megacarioblástica en la médula ósea

\begin{tabular}{lcc} 
Caso & Diagnóstico & Cariotipo \\
\hline 1 & MAT & $47, \mathrm{XY},+21 \mathrm{c}[20]$ \\
2 & MAT & $47, \mathrm{XX},+21 \mathrm{c}[20]$ \\
3 & MAT & $47, \mathrm{XY},+21 \mathrm{c}[20]$ \\
4 & MAT & $46, \mathrm{XY},-19,+21 \mathrm{c}[3] / 47, \mathrm{XY},+21 \mathrm{c}[14]$ \\
5 & MAT & $47, \mathrm{XX},+21 \mathrm{c}[20]$ \\
$6^{*}$ & MAT & $47, \mathrm{XY},+21 \mathrm{c}[20]$ \\
7 & MAT & $47, \mathrm{XY},+21 \mathrm{c}[20]$ \\
8 & MAT & $47, \mathrm{XY},+21 \mathrm{c}[20]$ \\
9 & MAT & $47, \mathrm{XY},+21 \mathrm{c}[20]$ \\
10 & MAT & $47, \mathrm{XX},+21 \mathrm{c}[20]$ \\
11 & LMCA & $46, \mathrm{XX},+21 \mathrm{c}, \mathrm{der}(21 ; 21)(\mathrm{q} 10 ; \mathrm{q} 10) \mathrm{c}[20]$ \\
$12^{*}$ & LMCA & $47, \mathrm{XY},-7,+21 \mathrm{c},+\operatorname{mar}[10] / 48, \mathrm{XY}, \mathrm{idem},+21[6] / 47, \mathrm{XY},+21 \mathrm{c}[4]$ \\
13 & LMCA & $47, \mathrm{XY}, \mathrm{i}(7)(\mathrm{q} 10),+21 \mathrm{c}[4] / 47, \mathrm{XY},+21 \mathrm{c}[16]$ \\
14 & LMCA & $46, \mathrm{XY}, \operatorname{der}(15) \mathrm{t}(9 ; 15)(\mathrm{q} 11 ; \mathrm{q} 11.2),-17, \operatorname{add}(19)(\mathrm{p} 13.3), \mathrm{der}(21) \mathrm{t}(17 ; 21)(\mathrm{q} 11.2 ; \mathrm{p} 13),+\operatorname{mar}[16] / 47, \mathrm{idem},+21 \mathrm{c}[4]$ \\
\hline
\end{tabular}

* Mismo paciente.

MAT: mielopoyesis anormal transitoria. LMCA: leucemia megacarioblástica aguda.

TABLA 3. Resumen de las mutaciones del gen GATA-1 en las muestras de médula ósea estudiadas y predicción de sus consecuencias a nivel proteico

\begin{tabular}{|c|c|c|c|c|}
\hline Caso & Diag. & Mutación* & Consecuencia de la mutación & Subtipo de mutación ${ }^{* *}$ \\
\hline 1 & MAT & c.214_218dup & Error en el corte y empalme & ES \\
\hline 2 & MAT & c. $5 \mathrm{~A}>\mathrm{G}$ & Glu2Gly & $\mathrm{NC}$ \\
\hline 3 & MAT & c.90_91delAG & (Val32Phe) +38 Stop & PTC 1-5' \\
\hline 4 & MAT & c. $220+1 G>A$ & Error en el corte y empalme & ES \\
\hline 5 & MAT & c.151_184dup & Tyr62Stop & PTC 1-5' \\
\hline $6^{\#}$ & MAT & c.150_184dup & Tyr62Stop & PTC 1-5' \\
\hline 7 & MAT & c. $219 A>G$ & Error en el corte y empalme & ES \\
\hline 8 & MAT & c.217_220del & Error en el corte y empalme & ES \\
\hline 9 & MAT & c. $220+2 \mathrm{~T}>\mathrm{C}$ & Error en el corte y empalme & ES \\
\hline 10 & MAT & c.169_184dup + c.184_185insG & Tyr62Trp + 142Stop & PTC 1-3' \\
\hline 11 & LMCA & c.149_183dup & Tyr62Arg & $\mathrm{NC}$ \\
\hline $12^{\#}$ & LMCA & c.150_184dup & Tyr62Stop & PTC 1-5' \\
\hline 13 & LMCA & WT & WT & $\mathrm{NC}$ \\
\hline 14 & LMCA & c. $220 \mathrm{G}>\mathrm{A}$ & Error en el corte y empalme & ES \\
\hline
\end{tabular}

* El nucleótido número 1 corresponde a la A del codón ATG de iniciación de la traducción ubicado en el exón 2 del gen GATA-1 de acuerdo con la secuencia de referencia (NM_002049.3).

** Clasificación según Kanezaki y cols. ES: error en el corte y empalme (splicing); NC: no corresponde.

\# Mismo paciente.

MAT: mielopoyesis anormal transitoria. LMCA: leucemia megacarioblástica aguda. 
hallaron nuevas alteraciones citogenéticas en el momento del diagnóstico de esta última.

Las mutaciones detectadas se describen en la Tabla 3, incluidas sus consecuencias a nivel proteico y su clasificación según lo propuesto por Kanezaki y cols. ${ }^{10}$

De las 13 mutaciones identificadas en las muestras de MAT y LMCA, 8 (62\%) correspondieron a inserciones /deleciones / duplicaciones y $5(38 \%)$, a sustituciones de bases. Se detectaron mutaciones en todos los pacientes con MAT; los tipos de mutaciones caracterizadas fueron duplicaciones $(n=5)$, sustituciones de nucleótido simple $(n=4)$ y deleción $(n=1)$. En 3 de los 4 pacientes con LMCA se evidenció la presencia de duplicaciones $(n=2)$ o sustitución de nucleótido simple $(n=1)$.

El análisis computacional de las consecuencias de las mutaciones a nivel proteico mostró la presencia de un codón de terminación prematuro de la traducción en 5 muestras $(\mathrm{MAT}=4 / \mathrm{LMCA}$ $=1$ ), la pérdida del exón 2 por modificaciones en los sitios de corte y empalme en 6 casos (MAT = 5/ LMCA $=1$ ) en los cuales las mutaciones afectaban sitios críticos de reconocimiento de la señal (Figura 1). En dos casos las mutaciones se tradujeron en cambio de la secuencia aminoacídica.

En la evolución de los 10 pacientes con MAT se observó que 8 alcanzaron la remisión completa en forma espontánea, sin recibir ningún tipo de quimioterapia. Un paciente murió antes de alcanzar la remisión completa debido a una infección respiratoria aguda y con un paciente se perdió el contacto en el seguimiento. De los 8 pacientes que alcanzaron la remisión

FIGURA 1. Esquema representativo del exón 2 del gen GATA-1, donde se señala la ubicación de los sitios de las mutaciones detectadas. El símbolo indica el tipo de consecuencia a nivel proteico. Pérdida del exón 2 por error en el corte y empalme, cambio en la secuencia aminoacídica, codón de terminación de la traducción prematuro en el exón 2 y codón de terminación de la traducción prematuro en el exón 3

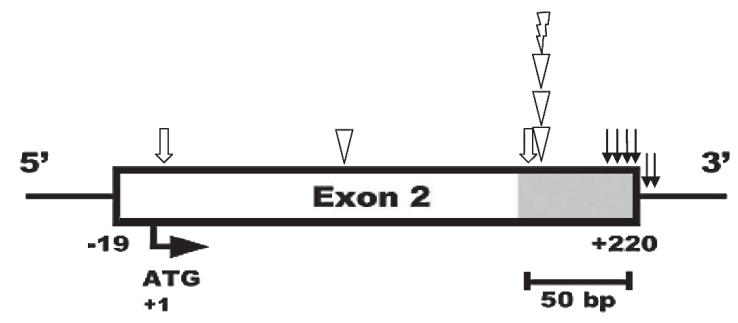

completa, 3 permanecen en remisión completa con $+48,+55$ y +90 meses de seguimiento. De los restantes 5 pacientes que alcanzaron la remisión completa, uno presentó LMCA después de 9 meses de remisión completa, 3 murieron en remisión completa debido a sepsis en 2 casos y a descompensación de la cardiopatía en el restante. Un paciente no concurre a control desde hace 31 meses.

En cuanto a los 4 pacientes con LMCA, todos alcanzaron la remisión completa. Uno de ellos, recayó tempranamente, se le administró un segundo tratamiento, pero murió debido a complicaciones infecciosas. Los restantes 3 pacientes viven en remisión completa a $+22,+46$ y +65 meses desde su diagnóstico.

\section{DISCUSIÓN}

Las patologías hematológicas asociadas a mutaciones del gen GATA-1 se presentan a temprana edad y, por ello, se especula que estas alteraciones se originarían intraútero, durante el desarrollo fetal. Esto coincide con la hepatomegalia observada en todos los casos de MAT analizados en el momento del diagnóstico, ya que el hígado cumple una función primordial durante la hematopoyesis fetal.

La presencia de la trisomía 21 se considera el primer evento o alteración genética, que predispone a las células hematopoyéticas a la adquisición de mutaciones en el gen GATA-1 que explicarían la aparición del síndrome de MAT, aunque se desconoce la fisiopatogenia de la remisión espontánea observada en estos casos.

Las mutaciones en GATA-1 se detectaron en todos los pacientes con diagnóstico de MAT analizados. Con respecto al paciente con diagnóstico de MAT que remitió espontáneamente y 9 meses más tarde presentó LMCA, si bien se comprobó la presencia de la misma mutación en GATA-1 en ambas muestras, el análisis citogenético muestra la adquisición de nuevas anomalías por parte del clon leucémico. Estos hallazgos fortalecen la teoría de que ambos cuadros involucran la misma población de células hematopoyéticas y sugiere que la evolución de MAT a LMCA podría ser la consecuencia de la adquisición de mutaciones adicionales.

Es importante considerar la edad de presentación del cuadro clínico, un dato relevante para realizar el diagnóstico diferencial entre MAT y LMCA, ya que el $80 \%$ de los casos de MAT se resuelven espontáneamente antes de los 3 primeros meses de vida sin necesidad de 
tratamiento. Si bien el diagnóstico de MAT se basa en las características clínicas y hematológicas del paciente, y fundamentalmente en la evolución a la remisión espontánea, la demostración de la presencia de mutaciones en el gen GATA-1 por técnicas de biología molecular podría utilizarse para orientar la sospecha diagnóstica en los pacientes con Síndrome de Down sin manifestaciones clínicas o hematológicas.

Algunos autores proponen la administración preventiva de bajas dosis de quimioterapia a los pacientes con MAT, ${ }^{11}$ pero siempre debe tenerse en cuenta la morbi-mortalidad de estos tratamientos en los recién nacidos con diagnóstico de Síndrome de Down y reservar el tratamiento para aquellos casos que presenten complicaciones clínicas potencialmente mortales.

Para nuestro conocimiento, este es el primer estudio realizado en la Argentina, en el que se caracterizaron las mutaciones del gen GATA-1 en pacientes con Síndrome de Down y diagnóstico de MAT y LMCA. La alta frecuencia de mutaciones encontrada en recién nacidos con Síndrome de Down y MAT sugeriría la conveniencia de investigar su presencia en todos los neonatos con Síndrome de Down a fin de individualizar a aquellos con mayor riesgo de presentar MAT o LMCA. Estos últimos pacientes deberían recibir un control clínico más riguroso que aquellos neonatos con Síndrome de Down que no las presentan, con la finalidad de realizar un diagnóstico precoz que evite las complicaciones del cuadro. Además, la evaluación seriada de las mutaciones detectadas permitiría monitorear a los pacientes con MAT para comprobar la cinética de desaparición del clon preleucémico y predecir tempranamente una eventual progresión a LMCA.

El presente trabajo ha permitido confirmar la alta frecuencia publicada para las mutaciones en el gen GATA-1 en los neonatos con Síndrome de Down y MAT o LMCA. ${ }^{8,10,12}$ El conocimiento de esas mutaciones contribuirá a una mejor comprensión de los mecanismos leucemogénicos asociados a estas patologías en los niños con Síndrome de Down, lo cual favorecerá la detección de potenciales blancos terapéuticos para futuras estrategias de tratamiento.

\section{BIBLIOGRAFÍA}

1. Fong CT, Brodeur GM. Down's syndrome and leukemia: epidemiology, genetics, cytogenetics and mechanisms of leukemogenesis. Cancer Genet Cytogenet 1987;28:55-76.

2. Webb D, Roberts I, Vyas P. Haematology of Down syndrome. Arch Dis Child Fetal Neonatal 2007;92:503-7.

3. Baumann I, Niemeyer CM, Brunning RD, Arber DA, et al. Myeloid proliferations related to Down Syndrome. En: Sdwerdlow SH, Campo E, Lee Harris N, Jaffe ES, eds. WHOClassification of Tumours of Haematopoietic and Lymphoid Tissues. Lyon, France: International Agency for Research on Cancer; 2008. Págs.142-4.

4. Cantor A, Orki S. Transcriptional regulation of erythropoiesis: an affair involving multiple partners. Oncogene 2002;21:3368-76.

5. Shivdasani RA. Molecular and transcriptionalregulation of megakaryocyte differentiation. Stem Cells 2001;19:397-407.

6. Wechsler J, Greene M, Mc Devitt MA, Anastasi J, et al. Acquired mutations in GATA1 in the megakaryoblastic leukemia of Down syndrome. Nat Genet 2002;32:148-52.

7. Ahmed M, Sternberg A, Hall G, Thomas A, et al. Natural history of GATA1 mutations in Down syndrome. Clinical observations, interventions, and therapeutic trials. Blood 2004;103:2480-9.

8. GroetJ,McElwaineS,Spinelli M, Rinaldiet A, etal. Acquired mutations in GATA1 in neonates with Down's syndrome with transient myeloid disorder. Lancet 2003;361:1617-20.

9. Hitzler JK, Cheung J, Li Y,SchererSW,Zipursky A. GATA1 mutations in transientleukemia and acutemegakaryoblastic leukemia of Down syndrome. Blood 2003;101:4301-4.

10. KanezakiR, TokiT, Terui K, XuG, etal. Down syndrome and GATA1 mutations in transient abnormal myeloproliferative disorder: mutation classes correlate with progression to myeloid leukemia. Blood 2010;116:4631-8.

11. Al-Ahmari A, Shah N, Sung L, Zipursky A, et al. Long-term results of an ultra low-dose cytarabine-based regimen for the treatment of acute megakaryoblastic leukaemia in children with Down síndrome. Brit J Haematol 2006;133:646-8.

12. Alford KA, Reinhardt K, Garnett C, Norton A, et al. Analysis of GATA1 mutations in Down syndrome transient myeloproliferative disorder and myeloid leukemia. Blood 2011;118:2222-38. 